\title{
Progress with Scale-Up of HIV Viral Load Monitoring — Seven Sub-Saharan African Countries, January 2015-June 2016
}

\begin{abstract}
Shirley Lecher, $\mathrm{MD}^{1}$; Jason Williams, $\mathrm{MPH}^{2}$; Peter N. Fonjungo, $\mathrm{PhD}^{1}$; Andrea A. Kim, $\mathrm{PhD}^{1}$; Dennis Ellenberger, $\mathrm{PhD}^{1}$; Guoqing Zhang, PhD ${ }^{1}$; Christiane Adje Toure, $\mathrm{PhD}^{3}$; Simon Agolory, $\mathrm{MD}^{4}$; Georgette Appiah-Pippim, MD ${ }^{1}$; Suzanne Beard, $\mathrm{PhD}^{1}$; Marie Yolande Borget ${ }^{3}$;

Sergio Carmona, $\mathrm{MBBCh}^{5}$; Geoffrey Chipungu, $\mathrm{MBBS}^{6}$; Karidia Diallo, $\mathrm{PhD}^{1}$; Marie Downer, $\mathrm{MD}^{7}$; Dianna Edgil, $\mathrm{PhD}^{2}$; Holly Haberman, $\mathrm{MD}^{1}$; Mackenzie Hurlston, $\mathrm{MPH}^{1}$; Steven Jadzak ${ }^{1}$; Charles Kiyaga, $\mathrm{MSc}^{8}$; William MacLeod, ScD ${ }^{9}$; Boniface Makumb, MD ${ }^{10}$; Hellen Muttai, MBChB ${ }^{7}$; Christina Mwangi, MMed ${ }^{11}$; Jane W. Mwangi, MBChB 7 ; Michael Mwasekaga ${ }^{12}$; Mary Naluguza, PhD ${ }^{11}$; Lucy W. Ng'Ang'A, MBChB ${ }^{7}$; Shon Nguyen, MPH ${ }^{1}$; Souleymane Sawadogo, $\mathrm{PhD}^{4}$; Katrina Sleeman, $\mathrm{PhD}^{1}$; Wendy Stevens, MBBCh${ }^{5}$; Joel Kuritsky, $\mathrm{MD}^{2}$; Shannon Hader, MD ${ }^{1}$; John Nkengasong, PhD ${ }^{1}$
\end{abstract}

The World Health Organization (WHO) recommends viral load testing as the preferred method for monitoring the clinical response of patients with human immunodeficiency virus (HIV) infection to antiretroviral therapy (ART) (1). Viral load monitoring of patients on ART helps ensure early diagnosis and confirmation of ART failure and enables clinicians to take an appropriate course of action for patient management. When viral suppression is achieved and maintained, HIV transmission is substantially decreased, as is HIV-associated morbidity and mortality (2). CDC and other U.S. government agencies and international partners are supporting multiple countries in sub-Saharan Africa to provide viral load testing of persons with HIV who are on ART. This report examines current capacity for viral load testing based on equipment provided by manufacturers and progress with viral load monitoring of patients on ART in seven sub-Saharan countries (Côte d'Ivoire, Kenya, Malawi, Namibia, South Africa, Tanzania, and Uganda) during January 2015-June 2016. By June 2016, based on the target numbers for viral load testing set by each country, adequate equipment capacity existed in all but one country. During 2015 , two countries tested $>85 \%$ of patients on ART (Namibia [91\%] and South Africa [87\%]); four countries tested $<25 \%$ of patients on ART. In 2015, viral suppression was $>80 \%$ among those patients who received a viral load test in all countries except Côte d'Ivoire. Sustained country commitment and a coordinated global effort is needed to reach the goal for viral load monitoring of all persons with HIV on ART.

The Joint United Nations Programme on HIV/Acquired Immune Deficiency Syndrome (UNAIDS) “90-90-90" goals are to increase to $90 \%$ by 2020 the proportions of 1 ) persons living with HIV infection who know their status, 2) persons living with HIV infection receiving ART, and 3) persons living with HIV infection on ART who have achieved viral suppression (3). Substantial progress has been made in initiating ART among HIV-infected persons. In 2015, an estimated 17 million persons with HIV accessed treatment compared with 7.5 million in 2010, with sub-Saharan Africa accounting for approximately two thirds (11.8 million) of the persons on ART (4). Despite progress in ART coverage, substantial challenges with access to viral load testing in resource-limited countries remain
$(4,5)$. Unlike western countries, which have relied on viral load testing to monitor virologic response to ART, low- and middleincome countries have historically relied on CD4 cell counts (which monitor immunologic improvement for patients on ART) because of the higher cost of viral load testing.

Challenges to viral load testing scale-up have been identified, including weaknesses in sample transport and laboratory workflow; finance and procurement; human resources (i.e., staffing shortages); laboratory equipment maintenance; and laboratoryclinic interfaces $(5,6)$. To address these barriers and increase access to viral load testing, the Diagnostic Access Initiative (which includes representatives from the U.S. President's Emergency Plan for AIDS Relief [PEPFAR], UNAIDS, WHO, and others), was launched to increase laboratory capacity and reduce pricing from manufacturers for better access to viral load testing (7). PEPFAR collaborates with manufacturers for procurement and viral loading testing reagents. CDC provides technical expertise in support of viral load testing, and the United States Agency for International Development (USAID) focuses on supply chain management, working with manufacturers for procurement and maintenance of viral load testing platforms. A previous evaluation found that countries were at various stages of implementation and that scale-up of viral load testing was feasible (G).

The seven sub-Saharan African countries were selected based on availability of data and agreements with ministries of health for January 2015-June 2016. Ministries of Health, CDC, and USAID program officers jointly collected information on viral load testing targets, viral load testing capacity, cumulative number of ART patients, number of ART patients with more than one viral load test, percentage of viral load tests indicating viral suppression $(<1,000$ virus copies/mL), test turnaround time, and number of CD 4 tests. The $\mathrm{WHO}$ algorithm for viral load testing suggests starting viral load testing 6 months after ART initiation, followed by viral load testing at 12 months if viral suppression is achieved, and yearly thereafter (8). Each country set their viral load testing targets (i.e., the number of persons to receive viral load testing) based on several factors, including the number of persons with HIV on ART and the country's ability to increase viral load testing. Information 
on the number of testing platforms and the overall testing capacity for each country was provided by the manufacturers, Roche and Abbott. Among the seven countries participating in the CDC HIV-1 Viral Load Proficiency Testing program, 62 laboratories were enrolled.

By June 2016, 176 molecular testing platforms were in use within the seven countries; Roche accounted for 107 (61\%) and Abbott 69 (39\%). By June 2016, based on the target numbers for viral load testing set by each country, adequate equipment capacity resided in all but Uganda. However, three countries (Malawi, Tanzania, and Uganda) did not have the capacity to test all the persons with HIV currently on ART at least once per year (Table 1) (Table 2). Of the 62 laboratories enrolled in viral load proficiency testing, $92 \%$ passed proficiency testing; only a few laboratories in Malawi and one in Kenya did not pass (Table 1). During January-June 2016, the mean test turnaround time was $\leq 4$ days in Namibia and South Africa, but was 28-50 days in all other countries (Table 1). During January 2015-June 2016, turnaround times decreased in Kenya and Uganda, but increased in Côte d'Ivoire, Malawi, and Tanzania.

During January 2015-June 2016, the number of patients on ART increased in all countries, with the greatest proportional increase observed in Uganda (13\%) and the lowest in Malawi (2\%) and Tanzania (2\%) (Table 2). The percentage of ART patients with one or more viral load tests during 2015 varied substantially across countries. Two countries tested $>85 \%$ of patients on ART: Namibia (91\%) and South Africa (87\%). Four countries, tested $<25 \%$ of ART patients: Côte d'Ivoire (10\%), Malawi (19\%), Tanzania (5\%), and Uganda (23\%) (Table 2). During January-June 2016, all countries reported testing $<50 \%$ of patients. Viral suppression was $\geq 82 \%$ in all countries except Côte d'Ivoire (66\%) and Tanzania (72\%). During January 2015-June 2016, viral suppression rates decreased by $12 \%$ in Côte d'Ivoire and $16 \%$ in Tanzania. CD4 test data were available for five countries. Overall, during January 2014-June 2016, four countries had declines in CD4 testing volume; Côte d'Ivoire had increases (Table 2).

\section{Discussion}

Global support from international agencies and collaboration with manufacturers has led to four of seven countries in sub-Saharan Africa having adequate capacity to perform viral load testing of all persons with HIV on ART per WHO guidance. All countries increased the number of patients receiving

TABLE 1. Human immunodeficiency virus viral load testing capacity and quality monitoring indicators, by country — seven sub-Saharan African countries, January 2015-June 2016

\begin{tabular}{|c|c|c|c|c|c|c|c|c|c|}
\hline \multirow[b]{2}{*}{ Country } & \multicolumn{2}{|c|}{$\begin{array}{c}\text { Established target } \\
\text { no. of tests }\end{array}$} & \multirow{2}{*}{$\begin{array}{c}\begin{array}{c}\text { Equipment } \\
\text { capacity }\end{array} \\
2015 / 2016\end{array}$} & \multicolumn{2}{|c|}{$\begin{array}{l}\text { Molecular testing } \\
\text { platforms }\end{array}$} & \multicolumn{2}{|c|}{ Laboratory proficiency testing } & \multicolumn{2}{|c|}{$\begin{array}{l}\text { Mean turnaround time } \\
\text { (days) }\end{array}$} \\
\hline & 2015 & 2016 & & Roche & Abbott & No. participated & No. passed & 2015 & Jan-Jun 2016 \\
\hline Côte d'Ivoire & 0 & 102,967 & 399,168 & 22 & 0 & 3 & 3 & 17 & 50 \\
\hline Kenya & $1,200,000$ & $1,393,557$ & $1,464,372$ & 15 & 21 & 16 & 15 & 48 & 36 \\
\hline Malawi ${ }^{\dagger}$ & 166,652 & 237,815 & 391,608 & 0 & 14 & 15 & 11 & 31 & 42 \\
\hline Namibia & 190,382 & 211,394 & 309,960 & 6 & 2 & 0 & 0 & 5 & 4 \\
\hline South Africa & $3,600,000$ & $3,900,000$ & $5,973.912$ & 37 & 14 & 16 & 16 & 3 & 3 \\
\hline Tanzania & 87,589 & 207,277 & 412,776 & 9 & 8 & 8 & 8 & 30 & 34 \\
\hline Uganda & 200,000 & 800,000 & 739,368 & 18 & 10 & 4 & 4 & 43 & 28 \\
\hline
\end{tabular}

* Mean turnaround time from whole blood draw to report sent from testing laboratory.

† Malawi guidelines recommend viral load testing every other year for persons on antiretroviral therapy.

TABLE 2. Human immunodeficiency virus treatment monitoring indicators, by country - seven sub-Saharan African countries, January 2015-June 2016

\begin{tabular}{|c|c|c|c|c|c|c|c|c|c|c|c|}
\hline \multirow[b]{2}{*}{ Country } & \multicolumn{2}{|c|}{ Total no. of ART patients } & \multicolumn{2}{|c|}{$\begin{array}{l}\text { No. of ART patients with } \\
\geq 1 \text { viral load test }\end{array}$} & \multicolumn{2}{|c|}{$\begin{array}{c}\% \text { ART patients with } \\
\geq 1 \text { viral load test }\end{array}$} & \multicolumn{2}{|c|}{$\begin{array}{c}\text { \% viral load tests } \\
\text { with viral } \\
\text { suppression* }\end{array}$} & \multicolumn{3}{|c|}{ No. CD4 tests } \\
\hline & 2015 & 2016 & 2015 & 2016 & 2015 & 2016 & 2015 & 2016 & 2014 & 2015 & 2016 \\
\hline Côte d'Ivoire & 147,947 & 160,561 & 15,502 & 17,114 & 10 & 11 & 78 & 66 & 186,159 & 145,755 & 177,815 \\
\hline Kenya & 860,297 & 923,000 & 650,645 & 456,756 & 76 & 49 & 83 & 84 & ND & ND & ND \\
\hline Malawi ${ }^{\dagger}$ & 595,186 & $606,673^{\S}$ & 115,971 & 115,528 & 19 & 19 & 82 & 89 & 125,543 & 75,973 & 16,164 \\
\hline Namibia & 143,805 & 148,940 & 130,367 & 63,732 & 91 & 43 & 87 & 87 & 50,091 & 23,424 & 8,048 \\
\hline South Africa & $3,318,384$ & $3,422,724$ & $2,875,734$ & $3,125,011$ & 87 & 91 & 83 & 83 & $3,933,588$ & $3,627,960$ & $1,736,211$ \\
\hline Tanzania & 758,344 & 769,527 & 41,289 & 66,344 & 5 & 9 & 88 & 72 & ND & ND & ND \\
\hline Uganda & $1,066,519$ & $1,213,091$ & 243,099 & 267,140 & 23 & 22 & 91 & 92 & $1,097,691$ & 960,241 & 341,019 \\
\hline
\end{tabular}

Abbreviations: $\mathrm{ART}=$ antiretroviral therapy; $\mathrm{ND}=$ data not available.

* Suppression $=<1,000$ copies $/ \mathrm{mL}$.

† Malawi guidelines recommend viral load testing every other year for persons on ART.

$\S$ These numbers reflect reported numbers from January-March 2016. 
ART, thus creating a greater demand for viral load testing. This increase is expected to continue as countries strive to reach the goal of initiating ART for all persons with HIV. Although the number of patients who have received viral load testing has increased, the percentage of patients on ART with at least one viral load test was $\leq 25 \%$ for four of the seven countries. In contrast, Namibia, Kenya, and South Africa tested $>75 \%$ of patients on ART. Among the seven countries, Namibia is closest to reaching the third " 90 " UNAIDS goal (to increase to $90 \%$ the proportion of persons with HIV infection on ART who have achieved viral suppression), followed by South Africa and Kenya. The remaining countries will need to make substantial progress to meet this goal.

Since 2014, the landscape for HIV patient monitoring has changed. CD4 testing is declining and viral load testing is increasing. This change is expected to continue as countries with limited resources prioritize monitoring efficacy of ART to reach viral suppression goals.

During January-June 2016, the turnaround time from specimen collection to return of results was 28-50 days in five countries, compared with $\leq 4$ days in two countries (Namibia and South Africa). Optimally, turnaround time would be $\leq 2$ weeks. Reasons for long turnaround time included equipment breakdown, reagent stock depletion, inefficient systems for specimen transport, and personnel shortages. Prolonged turnaround times can delay the prompt use of results for patient management, such as intense adherence counseling or switching to second-line ART, both of which improve viral load suppression. Substantially decreasing turnaround time is a factor that needs more focused attention in many countries.

The findings in this report are subject to at least three limitations. First, capacity for viral load testing was calculated using the optimal number of specimens that could be run on a machine based on guidance from manufacturers. Second, field conditions such as staffing shortages and power outages might decrease actual capacity. Finally, low viral load suppression rates might be the result of targeted testing for suspected treatment failure rather than unbiased testing of all persons with HIV on ART.

Successful scale-up of HIV viral load testing requires a global response. Because of coordinated efforts, millions of persons with HIV receive viral load testing despite limited resources. PEPFAR works directly with ministries of health and implementing partners to support viral load scale-up through establishing and optimizing systems. CDC, USAID, and other U.S. agencies provide complementary activities in molecular diagnostics, management of supply chain, and building human capacity. WHO has developed guidelines for viral load testing. The Clinton HIV/AIDS Initiative supports

\section{Summary \\ What is already known about this topic?}

Persons with human immunodeficiency virus (HIV) who have viral suppression have improved health outcomes and a lower risk for transmitting HIV infection to others. Thus, viral load testing is recommended for monitoring patients with HIV receiving antiretroviral therapy (ART). Increasing and monitoring the capacity for viral load testing are important measures for global control of HIV, particularly in sub-Saharan Africa, which has the highest prevalence of HIV worldwide.

What is added by this report?

During 2015-2016, among the seven sub-Saharan African countries evaluated, the number of patients with HIV receiving ART increased. Four countries now have the capacity to perform viral load testing for all patients currently on HIV treatment. All seven countries increased testing capacity. However, the percentage of patients on HIV treatment who received viral load testing was $<25 \%$ for four countries.

What are the implications for public health practice?

Continued international collaborative initiatives are needed to increase capacity for viral load testing, as well as access of these services among patients on HIV treatment in sub-Saharan Africa.

creation of electronic dashboards for data management. Continued coordination and effective partnerships are necessary to provide a harmonized, comprehensive approach that can maximize efficiencies and strengthen health systems for effective viral load scale-up.

\footnotetext{
${ }^{1}$ Center for Global Health, Division of Global HIV/AIDS and Tuberculosis, CDC; ${ }^{2}$ U.S. Agency for International Development, Washington, D.C.; ${ }^{3}$ Center for Global Health, Division of Global HIV/AIDS and Tuberculosis, CDC, Abidjan, Côte d'Ivoire; ${ }^{4}$ Center for Global Health, Division of Global HIV/AIDS and Tuberculosis, CDC, Windhoek, Namibia; ${ }^{5}$ Department of Molecular Medicine and Haematology, National Health Laboratory Service, Johannesburg, South Africa; ${ }^{6}$ Center for Global Health, Division of Global HIV/AIDS and Tuberculosis, CDC, Lilongwe, Malawi; ${ }^{7}$ Center for Global Health, Division of Global HIV/AIDS and Tuberculosis, CDC, Nairobi, Kenya; ${ }^{8}$ Central Public Health Laboratories, Kampala, Uganda; ${ }^{9}$ Health Economics and Epidemiology Research Office, Department of Internal Medicine, School of Clinical Medicine, Faculty of Health Sciences, University of the Witwatersrand, Johannesburg, South Africa; ${ }^{10}$ Namibia Institute of Pathology, Windhoek, Namibia; ${ }^{11}$ Center for Global Health, Division of Global HIV/AIDS and Tuberculosis, CDC, Kampala, Uganda; ${ }^{12}$ Center for Global Health, Division of Global HIV/AIDS and Tuberculosis, CDC, Dar es Salaam, Tanzania.

Corresponding author: Shirley Lecher, slecher@cdc.gov, 404-639-6315.
}

\section{References}

1. World Health Organization. Consolidated guidelines on the use of antiretroviral drugs for treating and preventing HIV infection: recommendations for a public health approach. Geneva Switzerland: World Health Organization; 2013. http:// apps.who.int/iris/bitstream/10665/85321/1/9789241505727_eng.pdf

2. Cohen MS, Chen YQ, McCauley M, et al.; HPTN 052 Study Team. Prevention of HIV-1 infection with early antiretroviral therapy. N Engl J Med 2011;365:493-505. http://dx.doi.org/10.1056/NEJMoa1105243 
3. Joint United Nations Programme on HIV/AIDS. 90-90-90: a transformative agenda to leave no one behind. Geneva, Switzerland: Joint United Nations Programme on HIV/AIDS; 2014. http://www.unaids.org/en/resources/ presscentre/unaidsspeeches/2014/20141025_SP_EXD_Vietnam_launch_ of_909090_en.pdf

4. Joint United Nations Programme on HIV/AIDS. UNAIDS fact sheet 2016. Geneva, Switzerland: Joint United Nations Programme on HIV/ AIDS; 2016. http://www.unaids.org/sites/default/files/media_asset/ UNAIDS_FactSheet_en.pdf

5. Roberts T, Cohn J, Bonner K, Hargreaves S. Scale-up of routine viral load testing in resource-poor settings: current and future implementation challenges. Clin Infect Dis 2016;62:1043-8. http://dx.doi.org/10.1093/cid/ciw001
6. Lecher S, Ellenberger D, Kim AA, et al. Scale-up of HIV viral load monitoring-seven sub-Saharan African countries. MMWR Morb Mortal Wkly Rep 2015;64:1287-90. http://dx.doi.org/10.15585/mmwr. mm6446a3

7. Peter T, Ellenberger D, Kim AA, et al. Early antiretroviral therapy initiation: access and equity of viral load testing for HIV treatment monitoring. Lancet Infect Dis. E-pub October 20, 2016.

8. World Health Organization. Technical and operational considerations for implementing HIV viral load testing. Geneva, Switzerland: World Health Organization; 2014. http://who.int/hiv/pub/arv/ viral-load-testing-technical-update/en/ 